\title{
Polymerase chain reaction with two molecular targets in mucosal leishmaniasis' diagnosis: a validation study
}

\author{
Clemencia Ovalle Bracho+, Luisa Porras de Quintana, \\ Sandra Muvdi Arenas, Melania Rios Parra
}

\author{
Tropical Dermatology Research Group, National Institute of Dermatology, Centro Dermatológico Federico \\ Lleras Acosta, E.S.E., Bogotá, Colombia
}

\begin{abstract}
We validated the polymerase chain reaction $(P C R)$ with a composite reference standard in 61 patients clinically suspected of having mucosal leishmaniasis, 36 of which were cases and 25 non-cases according to this reference standard. Patient classification and test application were carried out independently by two blind observers. One pair of primers was used to amplify a fragment of $120 \mathrm{bp}$ in the conserved region of kDNA and another pair was used to amplify the internal transcript spacers (ITS) rDNA. PCR showed $68.6 \%(95 \%$ CI 59.272.6) sensitivity and 92\% (95\% CI 78.9-97.7) specificity; positive likelihood ratio: 8.6 (95\% CI 2.8-31.3) and negative likelihood ratio: 0.3 (95\% CI 0.3-0.5), when kDNA molecular target was amplified. The test performed better on sensitivity using this target compared to the ITS rDNA molecular target which showed 40\% (95\% CI 31.5-42.3) sensitivity and 96\% (95\% CI 84.1-99.3) specificity; positive likelihood ratio: $10195 \%$ CI 2.0-58.8) and negative likelihood ratio: 0.6 (95\% CI 0.6-0.8). The inter-observer agreement was excellent for both tests. Based upon results obtained and due to low performance of conventional methods for diagnosing mucosal leishmaniasis, we consider PCR with kDNA as molecular target is a useful diagnostic test and the ITS rDNA molecular target is useful when the aim is to identify species.
\end{abstract}

Key words: leishmaniasis mucocutaneous - diagnosis - polymerase chain reaction - ribosomal spacer

Leishmaniasis is a disease found throughout the world: 350 million people are at risk, and the disease burden is 2.4 DALYs (years of life lost due to premature death or disability). It is estimated that there are 12 million cases in the world: 1.5 to 2 million new cases a year of which 1-1.5 million correspond to cutaneous leishmaniasis and 500,000 to visceral leishmaniasis (TDR/ WHO 2005). The total leishmaniasis cases reported in Colombia in 2005 were 18,100 showing a clear tendency for an increase in the last decade. This number represents a $21.85 \%$ increase with respect to the total cases informed by 2004 . The incidence rate estimated for cutaneous leishmaniasis for 2005 was $14.6 \times 10,000$, taking as denominator the rural population (Instituto Nacional de Salud 2006).

Mucosal leishmaniasis is a chronic progressive disease, difficult to treat and diagnose which usually appears months or years after skin inoculation and may lead to destructive lesions of the upper respiratory tract. It can be accompanied by malnutrition when it causes dys-

Financial support: Centro Dermatológico Federico Lleras Acosta and Instituto Colombiano para el Desarrollo de la Ciencia y la Tecnología "Francisco José de Caldas" (Colciencias) grant 212012-16939

Corresponding author: docencia@dermatologia.gov.co

Received 19 June 2006

Accepted 24 April 2007 phagia and can lead to death due to pneumonia or asphyxia (Marsden 1985).

Clinical diagnosis is difficult because symptoms and signs can be confused with those of other granulomatous and malignant conditions (Rodríguez et al. 1994). Parasite identification is difficult and sensitivity reports vary with different techniques, from $0.2 \%$ with direct smear to $68.6 \%$ when various methods are used (Weigle et al. 1987, Zajtchuk et al. 1989, Schubach et al. 2001, Disch et al. 2005, Oliveira et al. 2005). In cases where there is a strong clinical suspicion and no parasite is isolated, treatment is prescribed with pentavalent antimony which is highly effective, but has been associated with frequent adverse effects: malaise, headache, muscle pain, joint pain and hepatic, pancreatic, renal, hematologic, and cardiac toxicity that has lead to death in some cases (Chulay et al. 1985, Hepburn et al. 1994, Aronson et al. 1998).

Polymerase chain reaction (PCR) has been used in the last decade for cutaneous and more recently for mucosal leishmaniasis. Different authors have reported sensitivities that vary from 62 to $97.1 \%$ using mostly minicircle kDNA as target in mucosal leishmaniasis (Pirmez et al. 1999, Oddone et al. 2004, Oliveira et al. 2005, Disch et al. 2005). After carrying out a critical appraisal of these studies, we found that in most of them the measurements were not blind, the patient spectrum was not described, and the sample size ranged from 6 to 40 patients. The reference standard was not clearly stated in most, and varied in different articles, some used only clinical suspicion for case definition. There has been no previous study rigorously designed to validate PCR in the diagnosis of mucosal leishmaniasis, which was the main purpose of our research. 


\section{MATERIALS AND METHODS}

Study design - Diagnostic test validation study.

Eligibility criteria - Tissue samples used in this study were obtained from the tissue bank from the National Institute of Dermatology Centro Dermatológico Federico Lleras Acosta, Bogotá. The tissue samples used in this study belonged to patients that had a clinical diagnosis of leishmaniasis with suspicion of being the mucosal type. Samples from the tissue bank were used if complete clinical records were available, and if the DNA was of good quality.

Samples - Sixty-one biopsy samples were included: 52 from patients with clinical diagnosis of leishmaniasis with suspicion of being the mucosal type and 9 mucosal samples from individuals submitted to rhinoplasty.

The nasal samples were collected by an otorhinolaryngologist or a trained dermatologist using the following methodology: nasal mucosa was sprayed with lidocaine $1 \%$ and then cleaned with a saline solution and clorhexidine solution. After examination with a rhinoscope, the site with evident mucosal infiltration was identified. Then, 2\% lidocaine was injected in the selected site and a biopsy sample was taken with a nasal biopsy forceps. The biopsy samples were divided in two: one part was preserved in 10\% buffered formalin and processed for histopathologic examination, and the other part was flash frozen and stored at $-70^{\circ} \mathrm{C}$ until used in the PCR assay.

Reference standard - True positives were considered according to the below reference standard if the criteria 1 to 3 were met and at least one of the criteria 4 to 6 . The following criteria were considered for case definition: (1) clinical suspicion - the lesion was considered as suggestive of mucosal leishmaniasis if it had clinical infiltration associated with one or more symptoms and signs defined according to the anatomical area

TABLE I

Symptoms and signs according to area involved

\begin{tabular}{lll}
\hline Area involved & Symptoms & Signs \\
\hline Nasal & $\begin{array}{l}\text { Rhynorrea } \\
\text { Pruritus } \\
\text { Obstruction } \\
\text { Epystaxis }\end{array}$ & $\begin{array}{l}\text { Mucosal infiltration } \\
\text { Ulcer } \\
\text { Septum perforation } \\
\text { Cartilaginous septum } \\
\text { destruction }\end{array}$ \\
Oropharynx & $\begin{array}{l}\text { Odynophagia } \\
\text { Dysphagia }\end{array}$ & $\begin{array}{l}\text { Granulomatous infiltration } \\
\text { Uvula destruction } \\
\text { Fibrosis of the tonsilar bed }\end{array}$ \\
& Dysphonia & $\begin{array}{l}\text { Granulomatous infiltration } \\
\text { of laryngeal structures }\end{array}$ \\
& Doarse voice \\
Lip & Bleeding & Infiltration \\
Genitalia & Pain & Ulcer \\
& Pain & Mucosal infiltration \\
& Dysuria & Ulcer
\end{tabular}

involved (Table I); (2) histopathology findings - when either of these two categories were present in the biopsy samples: (a) when there was a pattern compatible or suggestive of mucosal leishmaniasis with dense and diffuse inflammatory infiltrate of plasma cells, lymphocytes, and macrophages, or isolated epithelioid cells and giant cells associated with the described inflammatory infiltrate or well circumscribed granulomas in the absence of amastigotes; (b) when amastigotes were identified associated with one of the inflammatory patterns previously described; (3) therapy test: when symptoms and signs were reduced one month after specific treatment with pentavalent antimony was begun; (4) Montenegro skin test (MST): considered positive if induration of $5 \mathrm{~mm}$ or greater was observed $48 \mathrm{~h}$ after antigen intradermal application; (5) scar: the presence or absence of a scar typical of cutaneous leishmaniasis was recorded; (6) history of living or having visited endemic areas before symptoms appeared.

Control patients (non-cases) were defined if one of the following criteria was fulfilled: (1) clinical suspicion of mucosal leishmaniasis, but a confirmed diagnosis of a different clinical entity; (2) clinical suspicion of mucosal leishmaniasis, a non specific inflammatory pattern not suggestive of mucosal leishmaniasis observed on histology, negative MST and clinical follow-up which showed no disease progression; (3) clinical diagnosis of a different mucosal disease confirmed by other diagnostic test.

In order to avoid interobserver variability that could be present if the histopathology reported in the patient's clinical record was used, each one of the histopathology slides was reviewed by one pathologist in order to classify each case in one of the described categories.

Although every case had an immunofluorescence test result, this criteria was not included in the reference standard because false positive results have been reported in Chagas disease, malaria, lupus erythematosus, and toxoplasmosis (Pappas et al. 1983).

DNA extraction - A positive control DNA was prepared from promastigotes of Leishmania braziliensis braziliensis strain MHOM/BR/75/M2903, and L. mexicana amazonensis reference strain IFLA/BR/67/PH8 which were grown in Schneider's insect medium. Positive control DNA and biopsy samples were immersed to complete lysis with $500 \mu \mathrm{l}$ of NET 10 solution $(\mathrm{NaCl} 5 \mathrm{M}$; buffer $10 \mathrm{mM}$ Tris- $\mathrm{HCl}$ (pH8.0) and $10 \mathrm{mM}$ EDTA; SDS $1 \%)$ and $20 \mu \mathrm{l}$ of proteinase $\mathrm{K}(20 \mathrm{mg} / \mathrm{ml})$ and heated at $56^{\circ} \mathrm{C}$ for $18 \mathrm{~h}$. The phenol-chloroform extraction protocol described by Isaza (2002) was used. The final DNA pellet was dissolved in $50 \mu \mathrm{l}$ of TE buffer $0.1 \times$ and stored at $-70^{\circ} \mathrm{C}$ until used. DNA quality was assessed through electrophoresis in agarose gel $0.8 \%$. DNA was considered to be of good quality if it showed a visible band of high molecular weight and no degradation. Samples that did not present these characteristics were excluded from the study.

Inhibition control - In order to detect reaction inhibitors, $2 \mathrm{ng}$ of $L$. b. braziliensis MHOM/BR/75/ M2903 DNA was added to one half of the reaction mixture. If this mixture, which contained both the patient's DNA and L. b. braziliensis DNA, did not reveal a visible 
band, it was interpreted as having inhibitors and a DNA purification protocol was used with $5 \mathrm{M}$ ammonium acetate (Sambrook et al. 2001). Once this procedure had been carried out, another PCR was performed in order to evidence the inhibitor removal.

$P C R$ - Each sample was analyzed using three different pairs of PCR primers: for ITS (internal transcript spacers) rDNA (Cupolillo et al. 1995), a 1000 bp sequence was amplified with IR1 (5' - GCT GTA GGT GAA CCT GCA GCA GCT GGA TCA TT - 3') and IR2 (5' GCG GGT AGT CCT GCC AAA CAC TCA GGT CTG 3'); for nested ITS PCR, primers ITS 1F (5' - GCA GCT GGA TCA TTT TCC - 3') and ITS 2R (5' - AAC ACT CAG GTC TGT AAA C - 3') were used. In order to amplify a $120 \mathrm{bp}$ sequence from the conserved region of kDNA (Rodgers et al. 1990), a modified 13A (5' - TAG GGG CGT TCT GCG AA - 3') and 13B (5' - ATT TTACAC CAA CCC CCA GTT - 3') primers were used. A PCR reaction mixture for ITS rDNA was prepared containing $1 \times$ amplification buffer $(20 \mathrm{mM}$ Tris-HCL pH 8,4 and $50 \mathrm{mM}$ $\mathrm{KCl}), 0,8 \mathrm{mM} \mathrm{MgCl}_{2}, 0,2 \mathrm{mM}$ of each dNTPs, $1 \mu \mathrm{M}$ of forward and reverse primer and 1.5 units of Taq polymerase in a final volume of $50 \mu \mathrm{l}$. Amplification conditions used for IR1-IR2 and ITS1F- ITS2R primers were as follows: samples were initially denatured at $94^{\circ} \mathrm{C}$ for 3 min, followed by 40 cycles of $94^{\circ} \mathrm{C}, 59^{\circ} \mathrm{C}$, and $72^{\circ} \mathrm{C}$ for $30 \mathrm{~s}$ each, with a final extension of $72^{\circ} \mathrm{C}$ for $10 \mathrm{~min}$. When using primers $13 \mathrm{~B}$ and modified $13 \mathrm{~A}$, reaction mixture was similar to the previous protocol except for $2 \mathrm{mM} \mathrm{MgCl}_{2}, 1.0 \mathrm{mM}$ dithiothreitol (DTT) and 1.25 units of Taq DNA polymerase (Invitrogen). Amplification conditions used were: samples were initially denatured at $94^{\circ} \mathrm{C}$ for $3 \mathrm{~min}$, followed by 35 cycles of $1 \mathrm{~min}$ each at $94^{\circ} \mathrm{C}, 60^{\circ} \mathrm{C}$, and $72^{\circ} \mathrm{C}$ respectively with a final extension for $10 \mathrm{~min}$ at $72^{\circ} \mathrm{C}$. Five microliters of DNA sample were added to final volume of $50 \mu \mathrm{l}$ for each reaction mixture. Each assay contained a negative control in which no DNA had been added to the reaction mixture and two positive controls in which $2 \mathrm{ng}$ of DNA from $L . b$. braziliensis MHOM/BR/75/M2903 DNA and L. $m$. amazonensis reference strain IFLA/BR/67/PH8 DNA had been included as a template for the PCR.

PCR product analysis - The PCR products were analyzed by electrophoresis in agarose gels of 1 and 2.5\% for $1000 \mathrm{pb}$ and $120 \mathrm{pb}$ respectively using Promega G2101 y G3161 size marker. Gels were visualized under ultraviolet light and photographed using Polaroid ${ }^{\mathrm{TM}}$ film. Interobserver agreement was measured for both molecular targets by two independent and blind observers who evaluated the presence or absence of the respective band on the gel for each sample.

Statistical analysis - Data obtained from clinical records for case definition was registered in a pre-designed form and entered in an Excel 2003 database. Statistical analysis was carried out with SPSS 11.5. Point estimates and $95 \%$ confidence intervales were estimated for: sensitivity, specificity, likelihood ratios, diagnostic odds ratio, kappa for inter and intra observer agreement using Javastat package.
Ethical considerations - This research was developed in accordance with the ethical standards established by the Institutional Ethical Committee of Centro Dermatológico Federico Lleras Acosta, which are based upon the Helsinki Declaration and was considered as less than minimal risk research.

\section{RESULTS}

Population characteristics - Fifty-two patients clinically suspected of having mucosal leishmaniasis and nine mucosal samples from individuals submitted to rhinoplasty entered the study. Thirty-six were classified as cases and 25 as non cases according to the reference standard used. The samples derived from individuals submitted to rhinoplasty were included as non cases. One hundred and eight eligible patients were excluded due to lack of information required for case definition in the clinical records or to inadequate biopsy samples or poor DNA quality. The mean age of the study subjects was 40.5 years and $65.6 \%$ were men. The range for evolution time was 1 to 25 years with a median of 18 months, and patients came from 10 different regions of the country. Patients classified as non cases were diagnosed with paracoccidioidomycoses, traumatic perforation, chronic cocaine or vasoconstrictor use, lepromatous leprosy, lymphoma, allergic rhinitis, and lupus. Eighteen percent of the non case group showed a positive MST. Among the group of patients classified as cases, $94 \%$ were MST positive and in 5\% of them parasites were identified in the histopathology. Eighty percent of cases showed a scar suggestive of cutaneous leishmaniasis. Indirect immunofluorescence was positive for $80 \%$ of cases, and negative for all controls.

Demographic characteristics' comparison between included and excluded individuals showed no significant differences: mean age difference was not statistically significant: $p=0.645$. There were no significant differences in gender proportions: $p=0.647$, and median time of evolution (months): $p=0.10$.

PCR leishmania detection - Twenty percent of the samples studied showed PCR inhibitors. After applying the DNA purification with 5M ammonium acetate only one showed persistent inhibition. DNA amplification with primer IR1-IR2 and ITS1F-ITS2R exhibited a $1000 \mathrm{bp}$ band (Fig. 1) and with primers modified 13A and 13B a 120 bp band was observed (Fig. 2). Table II shows the diagnostic operative test characteristics with a $95 \%$ confidence interval.

The two biopsy samples in which amastigotes were identified were positive for PCR using primers IR1-IR2, ITS1F-ITS2R, and modified 13A and $13 \mathrm{~B}$.

The inter-observer agreement was excellent; there were no discordant results for either test. The kappa statistic for kDNA was 1.0 (95\% CI 0.88-1.0) and for ITS rDNA was 1.0 (95\% CI 0.85-1.0)

Sixty eight percent of positive samples amplified DNA using primers B1 and B2 which are specific for braziliensis complex. Positive samples that did not amplify with these primers were analyzed using primers M1 and M2 (specific for mexicana complex) and were all negative. 
TABLE II

Diagnostic operative test characteristics

\begin{tabular}{lcccc}
\hline \multirow{2}{*}{ Operative characteristics } & \multicolumn{2}{c}{ ITS rDNA } & \multicolumn{2}{c}{ kDNA } \\
\cline { 2 - 5 } Sensitivity & $\%$ & $95 \%$ CI & $\%$ & $95 \%$ CI \\
Specificity & 40 & $31.5-42.3$ & 68.6 & $59.2-72.6$ \\
Positive predictive value & 96 & $84.1-99.3$ & 92 & $78.9-97.7$ \\
Negative predictive value & 93.3 & $73.4-98.8$ & 92.3 & $79.7-97.8$ \\
Likelihood ratio + & 53.3 & $46.7-55.2$ & 67.6 & $58-71.8$ \\
Likelihood ratio - & 10 & $2.0-58.8$ & 8.6 & $2.8-31.3$ \\
Diagnostic odds ratio & 0.6 & $0.6-0.8$ & 0.3 & $0.3-0.5$ \\
\hline
\end{tabular}

Sample with inhibition not included.

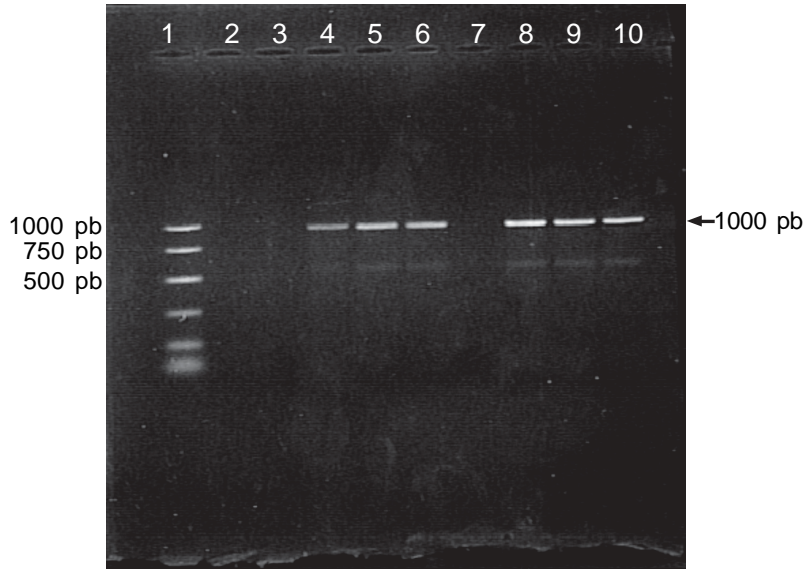

Fig. 1: polymerase chain reaction-internal transcript spacers (PCR-ITS) rDNA amplification for detection of Leishmania sp. Lines - 1: PCR markers G3161 (Promega Corp); 2: negative control; amplification using primers IR1-IR2; 3: non case; 4: inhibition control non case; 5: case; 6: inhibition control case, amplification using primers ITS1F - ITS2R; 7: non case; 8,9: cases; 10: positive control Leishmania braziliensis braziliensis (MHOM/BR/75/M2903).

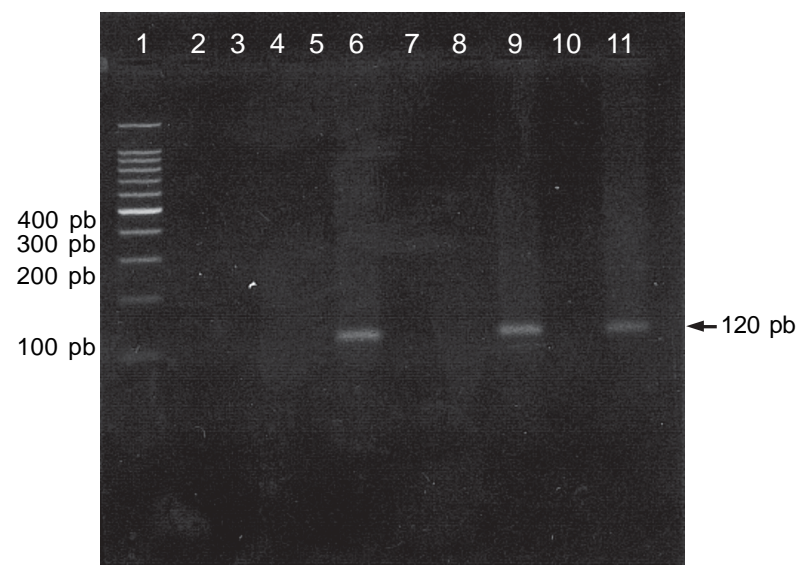

Fig. 2: polymerase chain reaction kDNA amplification for detection of Leishmania. Lines - 1: 100 pb DNA Ladder G2101 (Promega Corp); 2: negative control; 3, 4, 5, 7, 8, 10: non case; 6 , 9: case; 11: positive control Leishmania braziliensis braziliensis (MHOM/BR/75/M2903).

\section{DISCUSSION}

The aim of this study was to analyze the diagnostic operative characteristics of PCR using two molecular targets: conserved region kDNA and ITS rDNA in mucosal leishmaniasis. The reference standard used was a sum of clinical, epidemiological, and laboratory criteria, defined for this study.

Although the reference standard had not been used previously, we consider it had a good discrimination power. One could question the inclusion of individuals who showed a non-specific inflammatory infiltrate as controls, given the evidence of this type of infiltrate in leishmaniasis reported in the literature. Ridley described a series of over 400 biopsies of mucocutaneous leishmaniasis and found that non-specific inflammation was the most frequent and least effective response; its onset might be delayed, and in this event particularly the incidence of metastasis from skin to mucosa was high (Ridley 1989). This infiltrate can also be seen in individuals without the disease. In this work we considered individuals could be included as controls if the mucosal biopsy showed a non-specific inflammatory infiltrate and if they also had a negative MST and a follow up with no progressive course. Those patients included in our study as non cases had to have on the medical record a follow up were no disease progression was noted. If patients were lost, they were called and evaluated by researchers to determine if there was disease progression. We believe if the patient had a non specific infiltrate on mucosal biopsy, had a negative skin test, and was followed up in order to evaluate the disease did not have a progressive course without specific treatment, we could confidently classify this subject as a control and include him (her) in our study.

Studies designed to evaluate test diagnostic performance can have several biases which should be kept in mind when analyzing the results. The exclusion of a high proportion of eligible patients from the study could have left out a population not representative of the initial one. This could introduce a selection bias which occurs when not every patient presenting clinically relevant conditions is included in a consecutive order and when the selection is not random (Lijmer et al. 1999). Demographic characteristics and evolution time of symptoms 
were compared between excluded and included individuals in order to assess differences that could have introduced a selection bias in the study. No statistically significant differences were found between these groups $(p>0.05)$. The inclusion of healthy mucosal samples could have introduced a spectrum bias and enhance PCR specificity. We analyzed confidence intervals for diagnostic operative characteristics including and excluding these samples and did not find any significant differences. Test revision bias, which occurs when the result for the reference standard is known when applying the index test, as well as diagnosis revision bias which occurs when the results of the index test are known when interpreting the reference standard, were avoided by blinding the observers to each of the tests. The decision to apply one test was independent from the result of the other test and there was only one reference standard applied to all cases and non cases. Although the ideal would have been prospective data collection, in Colombia economic and sociopolitical reasons make it difficult for patients from certain rural areas to reach urban centers for follow-up. This is a limitation for retrospective as well as for prospective study designs.

Our result for PCR sensitivity when conserved region kDNA was used, showed a higher value $69.6 \%$ (95\% CI 59.2-72.6\%) than when ITS rDNA was used as molecular target. This difference could be due to differences in the number of copies present in Leishmania: around 10,000 copies for kDNA and near 200 copies for ITS rDNA (Rodgers et al. 1990, Pirmez et al. 1999, Marfurt et al. 2003, Bensoussan et al. 2006). Also, differences in the detection limits for each molecular target have been reported. Primers 13A and 13B can amplify $0.1 \mathrm{fg}$ of $L$. mexicana and $10 \mathrm{fg}$ of $\mathrm{kDNA}$ of $L$. braziliensis (Rodgers et al. 1990). Other authors inform detection limits for 13A and 13B primers of $<0.001$ parasite/reaction and 0.2 parasite/reaction for ITS 1 (Marfurt et al. 2003, Schonian et al. 2003). It is possible that the small amount of parasites present in the affected mucosa could be under the detection limit for ITS rDNA but could be enough for kDNA.

Previous studies inform sensitivity values that range from $62-97.1 \%$ with no confidence intervals reported. The reference standard used by some authors is not well described and varies from one study to another, which makes it difficult to establish comparisons. Most of the studies report case series with PCR made by non blind observers. None of the reports estimate inter-observer agreement (Pirmez et al. 1999, Medeiros et al. 2002, Oddone et al. 2004, Disch et al. 2005).

Sensitivity values in our report may have been affected by inhibitors, which were present in $20 \%$ of evaluated samples. The presence of contaminants in tissue samples that could inhibit Taq polymerase activity and reduce the sensitivity 10 to 100 times when compared with kDNA obtained from cultures has been described (Rodgers et al. 1990). The procedure used for DNA purification can reduce DNA quantity and increase the number of false negatives. Hemoglobin could be an inhibitor in these samples, but there may also be elements present in nasal mucus that can inhibit PCR reaction. The presence of inhibitors in 30\% of samples from nasal swabs of leprosy patients has been reported (Pattyn et al.1993).

The specificity reported was good for both molecular targets, so if a test is positive one can be highly confident that the diagnosis is mucosal leishmaniasis. One of the false positive results we obtained was positive for both molecular targets and we believe could have been misinterpreted by the reference standard. Previous reports have informed $100 \%$ specificity with a poor description of the population included as non cases.

Likelihood ratios for positive results were good and similar for both tests, although the confidence intervals were wide. For ITS rDNA there would be 10 positive results in mucosal leishmaniasis patients for each positive result in a patient with another condition. Likelihood ratios for negative results were also similar for both tests.

The diagnostic odds ratio, which expresses a ratio of the odds of a positive test in diseased and the odds of a positive test in non diseased is a measurement of the discrimination ability of the test and was good for both molecular targets, even though the precision was low due to a small sample size (Lijmer et al. 1999).

We consider our study has some strengths as well as some weaknesses. The strengths were the inclusion of a reasonable spectrum of individuals, the use of a composite reference standard, the independency of PCR in relation to the reference standard, and the blind interpretation of PCR and the reference standard. Among the weaknesses we can mention that the reference standard had not been used previously in the literature, the retrospective data collection and the exclusion of a high proportion of eligible subjects. We analyzed possible bias the study could have and could not identify any.

Considering the difficulty in identifying parasites from mucosal leishmaniasis lesions, we consider PCR a useful tool in the diagnosis of this clinical entity. When clinically suspected, PCR with kDNA as molecular target should be used due to its higher sensitivity. PCR ITS rDNA remains a useful test as it allows species identification.

Researchers should refine this technique in order to remove inhibitors without losing sensitivity. Multicenter prospective studies should be carried out in order to include a larger number of subjects and obtain more precise estimates. Well designed small studies could be useful for meta-analysis which address this subject.

\section{ACKNOWLEDGEMENTS}

To Martha Inirida Guerrero who was our scientific advisor and critically reviewed the manuscript, and Diana Alvarez for histopathology slides analysis.

\section{REFERENCES}

Aronson N, Glenn W, Johnson S, Jackson J, Gasser R, Magill A 1998. Safety and efficacy of intravenous sodium stibogluconate in the treatment of leishmaniasis: recent U.S. military experience. Clin Infect Dis 27: 1457-1464.

Bensoussan E, Nasereddin A, Jonas F, Schnur L, Jaffe L 2006. Comparison of PCR assays for diagnosis of cutaneous leishmaniasis. J Clin Microbiol 40: 1435-1439.

Chulay D, Effrey J, Spencer HC, Mugamei M 1985. Electrocardiographic changes during treatment of leishmaniasis with 
pentavalent antimony (sodium stibogluconate). Am J Trop Med Hyg 34: 702-709.

Cupolillo E, Grimaldi Jr G, Momen H, Beverley S 1995. Intergenic region typing (IRT): a rapid molecular approach to the characterization and evolution of Leishmania. Mol Bioch Parasitol 73: 145-155.

Disch J, Junqueira M, Orsini M, Pirmez C, Oliveira AC, Castro M, Rabello 2005. Leishmania (Viannia) subgenus kDNA amplification for the diagnosis of mucosal leishmaniasis. Diag Microbiol infect Dis 51: 185-190.

Hepburn NC, Siddique I, Howie AF, Beckett GJ, Hayes PC 1994. Hepatotoxicity of sodium stibogluconate therapy for American cutaneous leishmaniasis. Trans R Soc Trop Med Hyg 88: 453-455.

Instituto Nacional de Salud 2006. Informe de enfermedades transmitidas por vectores (ETV). Inf Quinc Epidem Nac 11: 40-44.

Isaza DM, Arboleda M, Restrepo M, McCann SHE, Barker DC 2002. Validation of the polymerase chain reaction for the diagnosis of human cutaneous leishmaniasis in north-west Colombia. Trans R Soc Trop Med Hyg 96: 165-168.

Lijmer J, Mol BW, Heisterkamp S, Bonsel G, Prins MH, van der Meulen JHP 1999. Empirical evidence of design-related bias in studies of diagnostic tests. JAMA 282: 1062-1066.

Marsden PD 1985. Clinical presentations of Leishmania braziliensis braziliensis. Parasitol Today 1: 129-133.

Medeiros A, Rodrigues S, Roselino A 2002. Comparison of the specificity of PCR and the histopathological detection of Leishmania for the diagnosis of American cutaneous leishmaniasis. Braz J Med Biol Res 35: 421-424.

Marfurt J, Nasereddin A, Niederwieser I, Jaffe CL, Beck H-P, Felger I 2003. Identification and differentiation of Leishmania species in clinical samples by PCR amplification of the miniexon sequence and subsequent restriction fragment length polymorphism analysis. J Clin Microbiol 41: 3147-3153.

Oddone R, Arbo C, Nara E, Velázquez GR, Acosta ME, Poletti D 2004. Utilidad diagnóstica de los métodos laboratoriales en leishmaniasis mucosa, incluyendo la "PCR". Noticias Técnicas del Laboratorio 4: 7-9.

Oliveira JG, Novail OF, Oliveira CI, Cruz JA, Campos FL, Rocjha A, Boaventura V, Noroña A, Jackson, ML Jackson, Barral A 2005. Polymerase chain reaction (PCR) is highly sensible for diagnosis of mucosal leishmaniasis. Acta Trop 94: 55-59.

Pappas Michael G, McGreevy PB, Hajkowski R 1983. Evaluation of promastigote and amastigote antigens in the indirect fluorescent antibody test for American cutaneous leishmaniasis. Am J Trop Med Hyg 32: 1260-1267.

Pattyn S, Ursi D, Ieven M, Grillone S, Raes V 1993. Detection of Mycobacterium leprae by the polymerase chain reaction in nasal swabs of leprosy patients and their contacts. Int $J$ Lepr 61: 389-393.

Pirmez C, Da Silva TV, Oliveira NMP, Da Cruz AM, Gonçalves CSC, Catanho M 1999. Use of PCR in diagnosis of human American tegumentary leishmaniasis in Rio de Janeiro, Brazil. J Clin Microbiol June: 1819-1823.

Ridley D, Magalhães A, Marsden P 1989. Histological analysis and the pathogenesis of mucocutaneous leishmaniasis. J Pathol 159: 293-299.

Rodgers MR, Popper SJ, Wirth DF1990. Amplification of Kinetoplat ADN as a tool in the detection and diagnosis of Leishmania. Exp Parasitol 71: 267-275.

Rodriguez N, Guzman B, Rodas A, Takiff H, Bloom BR, Convit J 1994. Diagnosis of cutaneous leishmaniasis and species discrimination of parasites by PCR and hibridization. J Clin Microbiol Sep: 2246-2252.

Sambrook J 2001. Molecular Cloning A Laboratory Manual. Preparation and Analysis of Eukaryotic Genomic DNA, 3rd ed., Cold Spring Harbor Laboratory Press, New York, p. 6.4-6.12

Schonian G, Nasereddin A, Dinse N, Schweynoch C, Schallig H, Presber W 2003. PCR diagnosis and characterization of Leishmania in local and imported clinical samples. Diag Microbiol and Infect Dis 47: 349-358.

Schubach A, Cuzzi-Maya T, Oliveira AV, Sartori A, De OliveiraNeto MP, Mattos MS 2001. Leishmanial antigens in the diagnosis of active lesions and ancient scars of American tegumentary leishmaniasis patients. Mem Inst Oswaldo Cruz. 96: 987-996.

Weigle KA, De Davalos M, Heredia P, Molineros R, Saravia NG, D'Alessandro A 1987. Diagnosis of cutaneous and mucocutaneous leishmaniasis in Colombia: a comparison of seven methods. Am J Trop Med Hyg 36: 489-496.

WHO-TDR 2005. Surveillance and control of leishmaniasis. http:/ /www.who.int/tdr/diseases/default.htm. Priorities and strategic emphases for TDR supported research on leishmaniasis. http://www.who.int/tdr/disease/leish/strategy.htm

Zajtchuk CJT, Casler JD, Netto EM, Grog M, Neafie RC, Hessel CR 1989. Mucosal leishmaniasis in Brazil. Laryngoscope 99: 925-939 